\title{
LE TOURISME ET LA FORÊT DANS LE NORD DE LA FRANCE
}

\author{
PAR \\ H. MESNIL \\ Conservateur des Eaux et Forêts \\ à Lille \\ et \\ R. MINAIR \\ Ingénieur des Travaux des Eaux et Forèts \\ à Valenciennes
}

La fréquentation de la forêt par les promeneurs est de tous les temps; par contre, l'afflux massif des populations dans les forêts suburbaines est récent. Avant 1914, les promenades sylvestres étaient réservées à ceux, relativement rares, disposant de loisirs. Il faut attendre 1936 et ses " congés payés ) pour voir la masse pénétrer en forêt. Actuellement, par suite de l'augmentation du parc automobile, le nombre de promeneurs se développe, chaque année, au point de créer de sérieux problèmes aux gestionnaires des massifs. Or, il faut résoudre ces problèmes, non dans un contexte purement administratif, mais dans un cadre humain, tenant compte des réalités sociales.

\section{L'attrait pour la forêt s'explique.}

Un effort méritoire a été fait pour accroitre le confort intérieur des logements, mais les grands ensembles ont une esthétique discutable, ils n'ont pas d'âme, ils ne retiennent pas; on est frappé de la monotonie des lignes droites, de leur parallélisme, de la symétrie des grands blocs. Des espaces dits "verts 》 ont été réservés pour supporter pelouses et plantations; l'idée est excellente, mais la réalité souvent plus décevante. Les arbres ont un aspect triste, accentué par la rareté de leurs feuilles: leur écorce, cible d'entraî- 
nement des jeunes possesseurs de canifs, pend en lambeaux lamentables. Quant au gazon des pelouses, il est usé par les ébats des enfants pour lesquels, trop souvent, rien n'a été prévu.

A cela s'ajoutent le roulement des camions, le hululement des sirènes, le brinquebalement des tramways, l'appel d'une locomotive, les cris des enfants, les impatiences des mères et, par-dessus tout, une lumière rare: brumes des Flandres ou du Hainaut accrochées aux terrils houillers, fumées des hauts fourneaux, condensations des centrales thermiques et ces mille émanations que l'industrie distille dans un ciel bas. Même par les plus beaux jours d'été, le ciel n'arrive pas à être bleu; il reste toujours accroché dans l'espace une transparence laiteuse donnant aux objets des contours sans netteté, et à l'âme une mélancolie certaine.

C'est dans ce décor que se réfugient, après une journée de travail, des hommes passant l'essentiel de leur vie à la mine, dans une usine, un bureau ou un magasin; piétinés, compressés dans les transports en commun, livrant une perpétuelle course contre la montre, ingérant à la hâte un repas à la cantine, ils se livrent en plus à 8,9 ou 10 heures de labeur, et quel labeur! Dans un bureau, le téléphone, le crépitement des machines à écrire et le souci de terminer la tâche avant le départ du courrier. A l'atelier, le bruit des. machines, le choc des marteaux sur la tôle, l'attention à soutenir, la chaleur à supporter, dans un courant d'air glacial, la poussière du charbon à 600 mètres sous terre. Etrange paradoxe: l'intellectuel est fatigué physiquement, et le manuel est surmené nerveusement!

Certes, l'expansion économique est un bienfait indiscutable hautement souhaitable, ne pouvant souffrir aucune limitation sous peine d'une décadence dont la nation tout entière pâtirait. Mais, en complément d'une civilisation en opposition avec le tempérament profond des hommes, est né un besoin d'évasion et de retour aux sources, d'autant plus fort que la plupart des citadins sont d'origine rurale. Or, c'est en forêt que se réalise le mieux ce retour à la terre; le calme, la solitude, la vie apparemment sauvage, l'indiscipline supposée des peuplements forestiers, attirent et reposent. La vie moderne dégrade la santé psychique. Les hommes souffrent d'être obligés de suivre une cadence trop brutale, et c'est inévitablement la lassitude, le ressort qui casse; une soirée de calme en forêt, le contraste des couleurs sous bois, lors d'une belle aprèsmidi d'automne, le renouveau d'une matinée de printemps, et la crise s'éloigne. La forêt contribue, à sa façon, à la lutte contre l'alcoolisme; après une journée sous bois, le consommateur de (" gros rouge ) ou de ("genièvre ) éprouve le besoin de boire de la limonade ou de la bière. Le camping débarrasse aussi l'homme de certaines habitudes d'intempérance. Le père de famille, après une journée de travail, retrouve la télévision, le dimanche, il va « aux sports » ou joue à la manille à l'estaminet, il voit ses enfants et 
sa femme à la hâte et chacun vit côte à côte, sans beaucoup se connaitre. Lors des promenades en forêt, il en est autrement: les enfants découvrent en leur père un compagnon de jeux, sachant les écouter et guider leurs conversations; le père et la mère, détendus, calmes, reprennent l'habitude de converser ensemble, c'est une nouvelle union familiale qui se crée ou se ressoude.

Ces avantages ne peuvent évidemment se chiffrer, mais, en fait, l'Etat, puissance publique, gardien de la société, et les grosses collectivités, en retirent une forte économie; par contre, l'Etat, propriétaire privé, voit, sinon ses revenus diminuer, tout au moins ses dépenses d'investissement augmenter; la balance reste, en fait, très positive. Dans l'hypothèse où les deux rôles se trouvent séparés, on peut admettre que les collectivités bénéficiaires du tourisme dédommagent le domaine privé en l'aidant dans son effort d'équipement, en consacrant à la forêt une fraction des économies réalisées sur le fonctionnement des hôpitaux et sur l'aide à l'enfance abandonnée; c'est du reste ce qu'ont parfaitement compris les Conseils Généraux du Nord et du Pas-de-Calais qui subventionnent les efforts entrepris par le Service Forestier pour l'aménagement touristique des forêts de la région.

Que faut-il entendre par tourisme forestier?

Pour l'hôtelier, le touriste, c'est le client qui vient vider son escarcelle dans la sienne. Peu importe qu'il s'agisse d'un promeneur ou d'un homme d'affaires.

Pour le fonctionnaire, le touriste est un ressortissant et sa définition ne dépasse pas les limites tracées par le Service.

Pour le forestier, le touriste, c'est l'individu pénétrant en forêt sans titre, c'est-à-dire tout le monde, à l'exception des forestiers, des adjudicataires de coupes, de chasse ou de pêche, des concessionnaires et de leurs employés ou ayants droit.

Le véritable touriste forestier est, à notre sens, celui qui vient en forêt pour y trouver un cadre capable de lui procurer des satisfactions intellectuelles ou physiques, poétiques ou artistiques, de nature à le reposer des sujétions de la vie courante et notamment de ses préoccupations professionnelles.

Néanmoins, tous les promeneurs répondant à cette définition ne pratiquent pas les mêmes formes de tourisme; leur mode de groupement, comme la durée de leurs déplacements, varient. Quelques jeunes viennent en forêt sans surveillance; on les voit, par exemple, au lendemain d'examens, évoluer sans guide et sans contrôle, par bandes. Ce défoulement prend, le plus souvent, des formes anarchiques, dont la forêt fait les frais. Fort heureusement, beaucoup de jeunes pénètrent en forêt en groupes organisés: voyages scolaires, groupes culturels, scouts, colonies de vacances. Ils retien- 
nent de leur fréquentation sylvestre un enseignement des plus profitables, surtout si celui-ci est pratiqué en liaison avec le Service Forestier local.

Mais, dans notre région, le tourisme familial est le plus représentatif. Il n'est pas rare de voir deux ou trois générations de la même famille, entassées dans la même voiture, avec le chien, les pliants, le jeu de boules; ils convergent, souvent avec plusieurs ménages amis, vers le même point, ils restent groupés près de l'auto, les jeux s'organisent, on pique-nique et on repart à la tombée de la nuit. Des milliers de voitures envahissent ainsi, chaque dimanche, les massifs. Même par temps maussade, la fréquentation est importante. Les jours de pointe sont le dimanche, le jeudi et le samedi, quelquefois le lundi, ainsi que les $1^{\text {er }}$ et 8 mai, journées du muguet. Ce tourisme, reflétant les habitudes sociales des Nordistes, est fort sympathique, il est touchant par bien des aspects, ne seraitce que par les preuves de sollicitude prodiguées à l'égard des vieillards, des enfants et des malades.

La fréquentation des massifs présente un autre caractère: celui du tourisme urbain de courte durée. Campeurs et caravaniers font les longs déplacements vers des régions plus ensoleillées, réservant les massifs du Nord pour les week-ends de printemps et d'arrière-saison. Quelques familles possèdent ou louent des maisons (lans les villages proches des forêts ou descendent dans les auberges forestières, mais pour la majorité, il ne s'agit que de promenades d'une journée, voire d'une après-midi, il s'ensuit que le nécessaire équipement en terrain de camping n'a pas besoin d'un grand développement.

En résumé, le tourisme forestier dans la région est familial, endimanché, motorisé, constant, mais de courte durée.

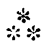

Le tourisme en forêt présente, entre autres, des inconvénients d'ordre sylvicole et cynégétique.

La foule des promeneurs accuse une inconscience totale. Il ne faut pas être surpris si une allumette ou un mégot sont jetés nonchalamment alors qu'ils ne sont pas éteints. Ce geste banal a pourtant les plus graves conséquences en période sèche dans les cantons envahis par la fougère ou dans ceux tapissés de molinie.

Si l'incendie reste le danger le plus grave, il n'est pas la seule cause de déprédation. Les possesseurs de petites maisons ont le désir légitime de planter quelques arbres autour de leur logis; aussi, la tentation est forte de prélever quelques “ sapins ) en forêt. Ces extractions délictueuses sont faites souvent en juillet, c'est-à-dire à une époque à laquelle la reprise est plus qu'aléatoire; elles se renouvellent, à l'occasion des fêtes de Noël. 
Un autre délit, en accroissement notable, est pratiqué par les femmes: elles n'hésitent pas à casser, au printemps, quelques mélèzes du Japon, le vert tendre des rosettes des jeunes aiguilles constituant un fond de bouquet idéal; en automne, les flammes des feuilles du chêne rouge attirent. Combien de jeunes plants n'ontils pas eu à souffrir de ces tailles barbares pratiquées par des mains élégantes! Le piétinement répété des talons à aiguilles a le même effet qu'un cylindre à pied de mouton et compacte le sol en un véritable béton.

Ces formes relativement récentes de déprédations s'ajoutent aux formes traditionnelles: initiales et cœurs gravés dans l'écorce des hêtres, jeunes perches transformées en balançoire, rameaux coupés pour l'édification d'une cabane.

Ces délits d'ordre sylvicole émeuvent, seulement, les forestiers. Il en va autrement lorsqu'il s'agit de délits intéressant les animaux de la forêt. Ceux-ci soulèvent fréquemment entre chasseurs et promeneurs des conflits aigus, voire violents. Le rôle d'arbitre que doit jouer, souvent, le Service Forestier, manque pour le moins de confort. Les chasseurs paient une location élevée. Ils consentent des dépenses importantes pour l'élevage du faisan, ils donnent des gages aux gardes-chasse, un salaire aux faisandiers. Chaque faisan porté au tableau revient, dans notre région, de 50 à 150 francs. On peut admettre que la susceptibilité des directeurs de chasse soit exacerbée par l'intrusion dans leur lot, de promeneurs qui, comme l'âne de la fable, se voient chargés par les gardes-chasse, de tous les péchés de la terre. A vrai dire, le promeneur n'est pas aussi malfaisant: les sangliers et les chevreuils ne sont pas peureux; le nombre de ces animaux tués chaque année sur les routes le prouve. En ce qui concerne les faisans, les dommages sont plus marqués, mais ils sont surtout le fait de braconniers organisés.

\section{$* * *$}

Les inconvénients très réels ainsi décrits posent des problènes.

Une attitude très administrative consiste à ne rien faire; il y a là pourtant un grave danger. Les touristes, même inorganisés, constituent une force, ils trouvent toujours une oreille complaisante, soit auprès de la presse, soit auprès des élus, pour y déposer leurs doléances. Toute limitation de leurs " droits ) est une offense personnelle. Si nous laissons cette force agir sans contrôle, le Service Forestier risque tôt ou tard d'être, sinon évincé de ses propres forêts, du moins mis en tutelle, soit qu'on lui impose de consentir à des déprédations exceptionnelles, soit qu'on le contraigne à un conservatisme exagéré. De toute façon, il perdra sa liberté de gestion et la production des massifs subira une régression importante allant jusqu'à mettre en cause la conservation de l'état boisé.

Le mouvement touristique forestier doit être guidé, ce qui suppose la satisfaction des besoins touristiques dans ce qu'ils ont de 
rationnel pour habituer nos interlocuteurs à demander plutôt qu'à se lancer dans des campagnes violentes et dangereuses. Il ne saurait pourtant être question de satisfaire les demandes sans établir entre elles une liaison suffisante que peut seul prévoir un plan préétabli en fonction d'une politique définie. Le plus souvent, le plan d'action s'inspire d'un texte, mais, en matière de tourisme forestier, il n'en existe pas, le code forestier étant muet à ce sujet. Or, la réglementation, même conçue à partir de principes excellents, devient rapidement une entrave pour tous. D'autre part, dans notre pays centralisé à outrance, il ne peut être pris que des textes d'application généralisés à tout le territoire qui, tenant compte de circonstances moyennes, sont toujours imparfaits sur le plan local. Enfin, au moment où tout se réglemente, se définit et s'intègre dans des formules, laissons au moins les loisirs s'épanouir dans la liberté, hors du cadre étroit où l'enfermeraient inévitablement des articles, même rédigés libéralement.

On peut songer à classer les peuplements en fonction de leur potentiel touristique. Nombre d'aménagements prévoient, déjà des séries touristiques où il est adopté un mode de traitement conçu spécialement pour la sauvegarde de certains points de vue, mais il est strictement impossible d'obliger les promeneurs à fréquenter ces séries à l'exclusion des autres: il faut rendre ces cantons accueillants, différents, pour que le public les préfère et renonce de plein gré au reste du massif. Or, accueil signifie équipement. Quant à la réglementation applicable à ces cantons, elle doit être envisagée sous une forme plutôt éducative que répressive.

L'origine germanique des populations de nos régions prédispose à une discipline spontanée, à peine atténuée par quinze siècles de culture latine et deux siècles d'occupation espagnole. De ce mélange d'influences est née une mentalité assez particulière et sympathique, qui se traduit par un besoin de discipline nuancée, mêlée d'une grande susceptibilité, quasi espagnole. Il suffit d'expliquer aux gens du Nord le sens d'un ordre, de les persuader du bien-fondé de cet ordre pour être sûr d'une obéissance complète, intelligente et même définitive. C'est là un terrain éminemment favorable pour tenter de remplacer des interdictions par une adhésion volontaire à des règles de courtoisie et de bienséance. Lorsqu'un promeneur pénètre en forêt, il s'insinue dans un ensemble complexe d'homme, d'animaux, de végétaux; il lui faut, sous peine de perturber le bon fonctionnement de cet ensemble, se soumettre à un certain nombre de règles; parmi celles-ci, quelques-unes se découvrent toutes seules, car elles relèvent de la plus élémentaire politesse, d'autres, au contraire, nécessitent des connaissances forestières. Cette éducation, c'est à nous, forestiers, à la faire.

Mais que dire, comment le dire, et par quels moyens?

Pour certains, éduquer signifie endoctriner. Or, le promeneur a ses préoccupations professionnelles dont il veut se reposer, il ne 
faut surtout pas l'accabler avec les nôtres. Il convient de rester sur un plan général et de vulgariser des idées paraissant évidentes; par la suite, certains interlocuteurs demanderont eux-mêmes à pénétrer dans la technique. Alors, ils constitueront un auditoire de choix, qui ne se lassera pas des subtilités de la sylviculture. Il faut commencer l'éducation du public par la vulgarisation d'idées très simples sur le respect de la nature végétale et animale en montrant, par exemple, que la forêt représente un équilibre souvent instable qu'il faut se garder de rompre. La forêt est utile à de multiples points de vue, il faut respecter les arbres, le sol, ne pas faire de feu. La forêt, c'est aussi un bel ensemble, animé par les animaux. Il faut éviter le mot " gibier ", il sonne mal à certaines oreilles et est souvent à l'origine de luttes sociales plus ou moins sourdes. Cette éducation doit se prolonger auprès des chasseurs et des gardeschasse, en leur faisant comprendre que leur intérêt bien compris est d'admettre les nécessités du tourisme.

Comment développer ces idées?

Il faut essayer de s'adresser au cœur comme à l'esprit, au sentiment comme au raisonnement, quelquefois même à l'instinct de possession, en faisant admettre que la forêt est le bien de tous. Enfin, l'éducation forestière doit avoir un caractère de permanence, d'où l'utilité des images faciles à retenir. Les spécialistes de la publicité le savent bien et se servent avec adresse de slogans astucieux, mille fois répétés. Comme on l'a dit fort justement " le Bon Dieu luimême a besoin de cloches pour se faire connaitre ").

Reste à trouver une tribune!

L'idéal serait sans doute l'enseignement des notions forestières, dans les écoles primaires, comme cela existe en Allemagne et dans la plupart des pays d'Europe Centrale. L'énumération des bienfaits de la forêt pourrait s'insérer aisément entre l'étude du bassin de la Loire et celle des conséquences de la Bataille de Bouvines. Cela n'existe pas, alors, à défaut de pouvoir atteindre les élèves, il faut parler aux maitres. Les conférences à l'Université, dans les écoles normales, dans les centres d'entrainement aux méthodes d'éducation active, doivent avoir les plus heureux effets; si on arrive à convaincre le futur maitre ou le moniteur, à travers lui et grâce à son rayonnement, combien de milliers d'enfants touchera$\mathrm{t}$-on? Le forestier dispose aussi d'autres tribunes: les Ecoles d'Agriculture, les C.E.T.A., les C.E.T.E.F., peuvent lui permettre d'aborder non seulement des aspects techniques, mais aussi des considérations plus vastes. Il faut surtout atteindre le grand public et, seuls, les moyens d'information classiques peuvent le permettre: la télévision, le cinéma, la radio, offrent des moyens de diffusion à utiliser largement, soit sur le plan national, soit sur le plan régional. Certaines émissions de télévision récentes ont fait un excellent travail en ce domaine. La presse écrite quotidienne ou périodique est 
aussi très utile par les communiqués qu'elle reproduit, par les articles de fond qu'elle consacre à tel ou tel aspect de la forêt et, parfois, par les relations qu'elle fait des événements de la vie forestière: congrès, tournées d'écoles, inaugurations, expositions forestières ou cynégétiques dans le cadre des foires, démonstrations de matériels forestiers, activités diverses, qui font penser à la forêt et à ses problèmes.

Mais c'est en forêt même que la vulgarisation revêt son aspect le plus concret: panneaux (" attention au feu ), affiches murales appliquées sur les maisons et les auberges forestières, prennent dans ce cadre une valeur d'actualité. Les fêtes sylvestres peuvent être le prétexte de banderolles sur lesquelles, après quelques mots bien sentis de bienvenue, les recommandations d'usage trouvent leur place. Les organisateurs peuvent être invités à diffuser, à intervalles réguliers, de courts communiqués de mise en garde, des voitures munies de haut-parleur jouent le même rôle. Cette vulgarisation globale doit être complétée par une vulgarisation individuelle. Un Agent Technique se présentant bien, s'exprimant sobrement, obtient des résultats de compréhension supérieurs à ceux obtenus par les moyens de diffusion classiques, mais il faut pour cela un certain entrainement psychologique: attendre les questions, rester simple et concret, n'aborder les détails techniques que sur demande.

Enfin, il y a le livre qui reste l'ami le plus sûr. Il est encore présent et disponible alors que les paroles sont envolées, et les journaux déchirés. Il existe des livres de vulgarisation remarquables, mais ils restent entre les mains d'initiés. Il leur faudrait une commune introduction sous forme, par exemple, d'une brochure largement distribuée. Les grandes entreprises ont des services de " $\mathrm{Pu}$ blic Relation ») et font paraître des ouvrages bien présentés et bien coloriés. Les adultes, surtout ceux qui se reposent, sont de grands enfants, aimant toujours les couleurs vives. Quelques dessins, même humoristiques, attireraient l'attention. Des slogans, imprimés en gros caractères, résumeraient le texte. Evidemment, de telles brochures coûteraient cher, mais ce serait de l'argent bien placé. A la longue, on aboutirait à une diminution des dépenses de défense préventive et de protection des forêts, et en tout cas, de réparation de dégâts.

L'éducation forestière, véritablement commencée depuis peu dans le Nord, y a déjà donné de bons résultats. L'Administration des Forêts est mieux connue; les rapports avec les autorités et les usagers de la forêt deviennent satisfaisants, souvent même cordiaux, mais la tâche est loin d'être achevée. Ce qu'il faut obtenir, c'est une armée d'amis de la forêt bien éduqués, bien guidés, dévoués, mais réalistes, car il existe des amoureux exclusifs plus dangereux que des ennemis. 
A ces promeneurs, il faut offrir un équipement touristique portant sur la route, le peuplement forestier, l'hôtellerie.

- Il existe souvent dans nos massifs un ensemble de routes forestières dont le tracé fait honneur à ses créateurs; il est très suffisant pour la vidange des coupes ou l'exercice de la chasse, c'està-dire pour le transport d' “ usagers ). Mais ce réseau, si bien conçu, souffre d'un mauviais entretien. On le comprend pour une région qui, en un quart de siècle, a vu les armées de deux continents parcourir les forêts, les bombarder, s'y retrancher, les raser. La pauvreté de la caisse des charges dans des massifs ayant payé un large tribut aux guerres successives ne permet même pas le comblement des nids de poule à la fourche... La dégradation est encore accentuée par un climat pluvieux qui délite les matériaux sur des chaussées mal aérées et assises sur des limons argileux. Le mauvais état des chemins était si général que, jusqu'en 1958, l'automobiliste arrivant à un carrefour forestier, pouvait prendre indifféremment l'une ou l'autre des routes s'ouvrant devant lui, aucun itinéraire n'offrant plus de confort qu'un autre. Par le jeu des digitations successives, on arrivait à la pénétration de la totalité des massifs sans profit pour personne. Le Service Forestier exerçait une surveillance illusoire, et l'on voyait çà et là, des touristes non contrôlés faisant une flambée près d'une jeune plantation. Les promeneurs n'y trouvaient pas non plus leur compte: les retours étaient maussades, les automobilistes, à la recherche d'un itinéraire perdu, visitaient chaque nid de poule, au grand dam de leurs passagers, comme de leur mécanique. Ils maudissaient l'Administration qu'ils jugeaient incapable. La remise en bon état d'un certain nombre de ces routes a, par contre, donné satisfaction à tous. On a assisté à un véritable cantonnement des promeneurs: l'automobiliste du dimanche qui passe deux heures à faire luire sa carrosserie avant et après chaque sortie a tout naturellement délaissé les mauvais chemins pour les bonnes routes. La longueur des itinéraires réfectionnés permet que ce cantonnement ne signifie pas concentration. Les agents techniques peuvent assurer une surveillance plus facile.

Le choix des itinéraires à remettre en état est essentiel. Dans la région, il a été basé sur un certain nombre de critères d'ordre plutôt " psychologique ». Tout d'abord, l'articulation du réseau rénové sur les routes publiques a toujours été recherchée. Des contacts avec les Ponts et Chaussées ont été pris, afin que notre effort ne reste pas isolé mais s'inscrive dans un plan d'ensemble. Ensuite, on recherche les routes qui, prenant leur départ à proximité des points d'accès les plus importants, sont nettement visibles des promeneurs et susceptibles de jouer un peu le rôle d'entonnoir. Enfin, on s'est efforcé de desservir tout ce qui peut être considéré 
comme point de sujétion: les sites recherchés, les terrains de camping, les maisons isolées, notamment, les maisons forestières, les points d'arrêt de la S.N.C.F. ou des lignes d'autọus, les auberges et établissements de cures, les réserves de chasse où il est possible de voir et photographier un chevreuil sautant un layon.

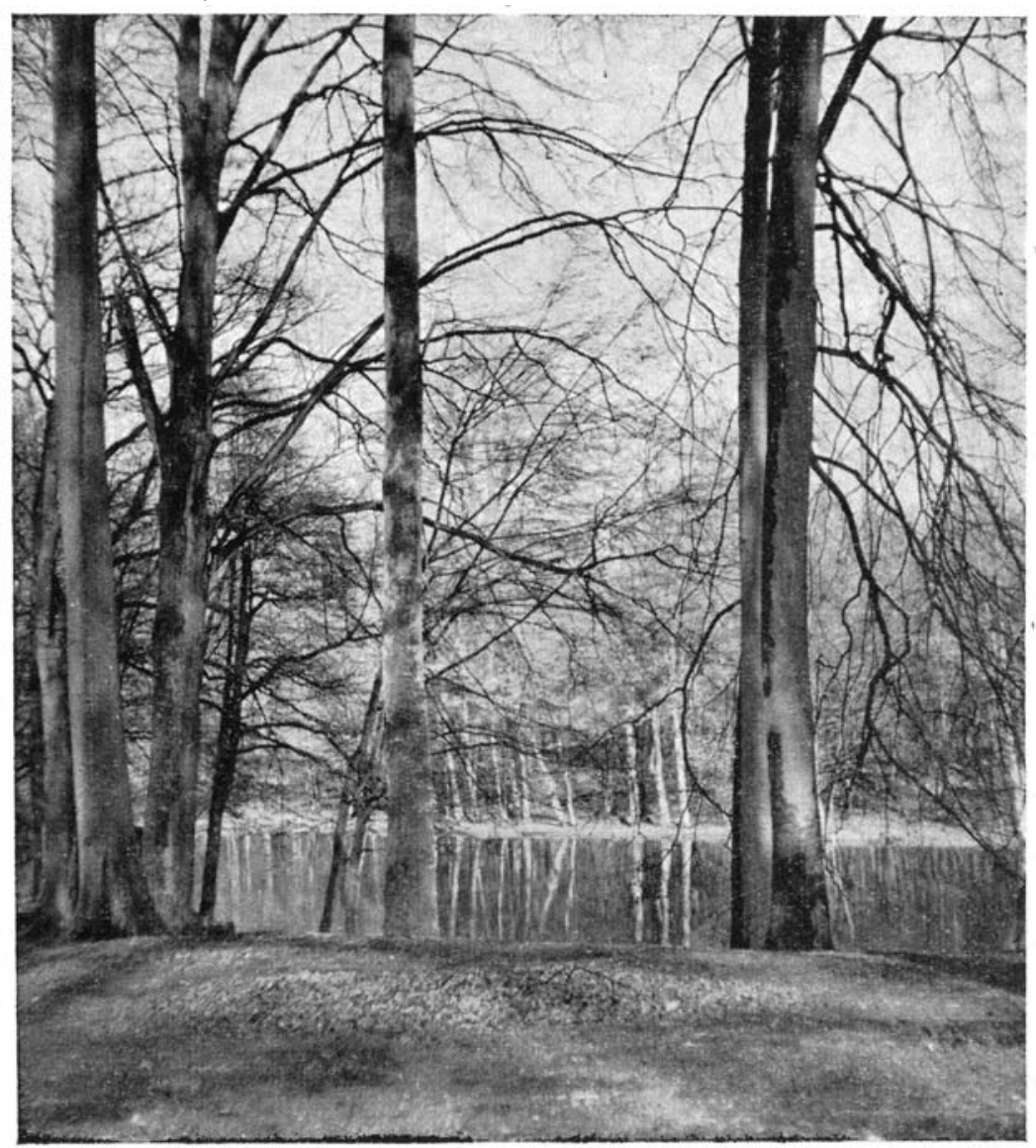

L'étang David, en forêt domaniale de Mormal

(Cliché Toulgouat.)

Le choix général étant fait, restait à fixer un ordre d'urgence, il fallait aller vite et commencer par la réfection des routes les moins coûteuses pour faire le plus de kilométrage possible. Après avoir paré au plus pressé, on pouvait envisager ensuite la remise en état des tronçons présentant des difficultés techniques plus grandes. Une 
largeur de 3,50 m en moyenne a été donnée aux routes rénovées; ceci pour deux raisons: la première parce que cette largeur correspond à l'utilisation la plus économique du finisher avec son maximum de deux rallonges, la seconde parce qu'il s'agit de routes de promenade, à circulation lente, permettant le croisement de deux véhicules de tourisme roulant à allure modérée. Plus large, il y aurait eu des courses de vitesse sur les tronçons rectilignes; or, il faut laisser au réseau public la circulation à grande vitesse et le transit des véhicules commerciaux. Au surplus, les routes à grand trafic fractionnent les massifs et rejettent les promeneurs vers d'autres cantons, au lieu de les attirer et de les retenir.

La technique routière est restée simple; c'est celle décrite dans. cette revue et qui a fait ses preuves, notamment en forêt de Compiègne. L'effort routier proprement dit est complété par la création de parkings. Suivant la fréquentation des routes, la conception de parking a été différente. Dans les forêts où l'on vient même par temps de pluie, les parkings sont " noirs », c'est-à-dire réalisés par semi-pénétration. C'est le cas de la forêt de Phalempin et de certains cantons de celle de Saint-Amand. Si la fréquentation est surtout intense par beau temps, les parkings " verts " ont été préférés: il s'agit alors de carrefours qui, après reprofilage au grader, s'enherbent et constituent, avec leur allure de gazon anglais, un point de stationnement idéal. Les. carrefours des forêts de Mormal et d'Hesdin ont été ainsi transformés en parkings verts. Enfin, à la limite, on a profité de la présence de terrils de schistes miniers de couleur rouge pour faire des " parkings rouges », qui, par le contraste des couleurs avec la route goudronnée, donnent un coup d'œil agréable. Ces parkings rouges sont nombreux en forêts de SaintAmand et de Marchiennes.

Le promeneur, souvent endimanché, ne tient pas à se déchirer aux ronces: il semble judicieux d'encourager les parcours pédestres sur des itinéraires spécialement aménagés en créant des sentiers de promenade interdits aux voitures; on évite ainsi l'intérieur des parcelles.

L'équipement a été poursuivi par la pose de corbeilles à papier. A vrai dire, les détritus sont souvent déposées au pied des corbeilles, mais certaines communes - Phalempin, Saint-Amand et Hesdin — procèdent régulièrement à l'enlèvement des ordures et on peut espérer qu'à la longue, on finira par obtenir une forêt propre.

Il reste encore à faire: outre que certains itinéraires, ayant un caractère touristique marqué, doivent être remis en état, il convient d'installer des bancs sur des emplacements favorables et d'aménager des allées cavalières à concéder aux Sociétés hippiques rurales, à charge par elles de les entretenir.

La circulation publique sur les routes forestières entraîne obligatoirement la responsabilité de l'Etat qui doit assurer la sécurité des automobilistes. Aussi l'autorité préfectorale a pris des arrêtés régle- 
mentant la circulation, qui ont donné toute satisfaction. Les agents techniques s'attachent à les faire respecter rigoureusement ce qui, non seulement dégage la responsabilité de l'Etat, mais impose aussi des prescriptions de nature à prévenir la dégradation des chaussées. Une signalisation adéquate matérialise l'arrêté préfectoral. En période de.dégel, des barrières blanches et rouges cadenassées, sont abaissées et supportent en leur milieu le disque réglementaire. Si on ajoute les rectangles ( attention au feu )) et les triangles (" attention gros gibier ) posés près des réserves de chasse, on arrive à une signalisation forestière complète susceptible d'assurer la sécurité des promeneurs.

L'équipement routier des forêts, commencé il y a quatre ans, porte sur un développement total de soixante-dix kilomètres dans le Nord et le Pas-de-Calais. Bien que non terminé, on peut admettre qu'il a donné une satisfaction presque complète à tous. Les automobilistes l'utilisent avec plaisir. Les journaux locaux publient périodiquement des articles élogieux pour l'Administration. Les chasseurs, réticents au départ, ont admis des routes facilitant leur déplacement, offrant des accotements débroussaillés où les jeunes faisans viennent se réchauffer; ils peuvent maintenant choisir des cantons peu parcourus pour en faire des zones d'élevage. En effet, l'intérieur des parcelles est peu fréquenté, une pénétration sur une bande de 50 mètres est un maximum. En ce qui concerne l'exploitation forestière, les difficultés de débardage restent les mêmes, mais les transports sur route sont facilités. Enfin, les liaisons internes sont aisées, que ce soit pour les interventions de sauveteurs en cas d'incendie, ou pour les tournées de service.

Les routes non goudronnées sont délaissées. Des disques (" sens interdit ) placés à 100 mètres de leur jonction avec les axes touristiques, les barrent; une surcharge " sauf autorisation ) empêche que la circulation des chasseurs et exploitants forestiers ne puisse être considérée comme un précédent. Les barrières à demeure sont rares, elles présentent le désavantage d'avoir un aspect vexatoire.. De toute façon, barrières ou disques sont presque inutiles, la peur des factures de garage constitue l'obstacle le plus sûr. Le macadam à l'eau, moins circulé, se repose, les arrachements de pierres, qu'une circulation intense de véhicules à pneumatiques provoquait, cessent. Et l'Etat ne risque plus le recours d'une compagnie d'assurances dont le client est allé au fossé pour éviter un nid de poule.

- Certes, l'objectif de production reste primordial, mais il devra concéder davantage aux nécessités touristiques. D’autre part, le touriste avouant avec candeur avoir admiré les résineux des Vosges ou du Jura, dédaigne les Pins sylvestres et les Epicéas du Nord. Il semblerait que le relief, le climat, la couleur du ciel, forment un cadre qui suppose telle essence plutôt que telle autre. Il a été préconisé, dans les directives d'aménagement, le retour pour les forêts 
touristiques, à l'éducation des hêtres. Lorsqu'on connaît l'enthousiasme soulevé par certains vieux peuplements, telle l'Allée de Hêtres en forêt de Saint-Amand, on ne peut qu'applaudir. Il semblerait que le vert sombre des feuilles charnues et le gris de l'écorce lisse des foyards, apportent dans ún paysage sans soleil, une douceur qui transforme en poésie ce qui ne serait que tristesse. Bien sûr, il faut cent vingt ans pour éduquer un hêtre, mais l'Etat, par la pérennité qu'il représente, n'a-t-il pas le droit ou même le devoir, de consentir les longues révolutions qu'on ne peut toujours conseiller aux particuliers?

- Lorsqu'on parle d'équipement hôtelier, il s'agit là d'un terme impropre, puisque dans notre esprit il groupe aussi bien terrains de camping et auberges forestières, mais il traduit une préoccupation d'accueil, quels que soient le mode de séjour, et sa durée.

Les collectivités locales et les grandes organisations de tourisme se sont efforcées d'offrir aux campeurs et aux caravaniers des terrains convenables. Un très beau terrain, avec théâtre de verdure, existe en forêt de Phalempin. La Municipalité de Saint-Amandles-Eaux aménage actuellement le terrain du Mont des Bruyères en forêt de Saint-Amand.

L'équipement commercial est plus développé. Nombre de brasseurs ou de propriétaires ont fait des efforts pour améliorer la présentation de leur commerce. Les forêts sont jalonnées d'auberges rustiques offrant la tarte de campagne, la bière, et le cidre de l'Avesnois, d'hostelleries bien achalandées, décorées avec goût, pour les promeneurs plus argentés. Aucune concurrence n'apporte de mésentente, chacun a sélectionné sa clientèle. I1 semblerait d'ailleurs, qu'au cours des années, cette clientèle s'affine, les clients les plus rudes deviennent courtois. On ne rencontre que des gens paisibles, tout est calme et poli, au sein d'une nature sereine.

Certes, ces considérations n'ont qu'un lointain rapport avec la foresterie, bien que l'Administration possède deux auberges en forêt de Mormal. Peut-on pourtant les négliger? Elles complètent notre équipement routier et sylvicole. Ces auberges forestières, ou les établissements de cure, comme celui de Saint-Amand-Thermal, donnent un cachet à la région, du pimpant à la forêt. Ce sont des points de fixation des promeneurs indispensables. Cela implique pour le Service Forestier, la nécessité d'encourager leur bonne présentation, de faciliter par des concessions rationnelles l'implantation de terrains de camping, de concourir par l'exemple à la relance d'une économie régionale touristique dont les citadins ont tant besoin.

En conclusion, on ne saurait trop insister sur l'importance des problèmes touristiques dans les forêts suburbaines du Nord, problèmes d'hier pour la région parisienne, d'aujourd'hui pour nous, 
de demain pour beaucoup, et qui bientôt - si ce n'est déjà fait feront éclater la traditionnelle classification des espaces boisés en forêts de production et en forêts de protection, pour y ajouter les forêts de " récréation ) d'où la production ne sera pas exclue si les forestiers savent prendre les devants. Et puis, il faut le dire, le tourisme éclairé, s'il sait conquérir un rang convenable dans l'ordre des priorités, sera un des éléments modérateurs des tentatives d'emprises industrielles, et contribuera à sauver les derniers lambeaux boisés qui subsistent encore, à proximité des centres à forte démographie. Le Service Forestier y trouvera, en fin de compte, une de ses raisons d'être et de subsister. 